\title{
Robust Taxi Dispatch under Model Uncertainties
}

Fei Miao

Shuo Han
Shan Lin

George J. Pappas

\begin{abstract}
In modern taxi networks, large amount of realtime taxi occupancy and location data are collected from networked in-vehicle sensors. They provide knowledge of system models on passenger demand and taxi supply for efficient dispatch control and coordinating strategies. Such dispatch approaches face a new challenge: how to deal with future customer demand uncertainties while fulfilling system's performance requirements, such as balancing service across the whole city and minimizing taxis' total idle cruising distance. To address this problem, we present a novel robust optimization method for taxis dispatch problems to consider polytope model uncertainties of highly spatiotemporally correlated demand and supply models. An objective function concave over the uncertain demand parameters and convex over the variables is formulated according to the design requirements. We transform the robust optimization problem to an equivalent convex optimization form by strong duality and minimax theorem, and computational tractability is guaranteed. By Monte-Carlo simulations, we show that the robust taxi dispatch solutions in this work are less probable to get large costs compared with non-robust results.
\end{abstract}

\section{INTRODUCTION}

Modern transportation systems equip various sensing technologies for passenger and vehicle tracking, such as global positioning system (GPS), radio-frequency identification (RFID), and occupancy sensing systems. Sensing data collected from transportation systems provides us opportunities for understanding spatiotemporal patterns of passenger demand. Researchers have developed methods to predict the probability distribution of taxi passenger demand [14], travel time [2], [10], [12], [17], and traveling speed [1].

Based on such rich spatiotemporal information about passenger mobility and demand, many control and coordination solutions have been designed for intelligent transportation systems, especially taxi networks. Geng and Cassandras design a smart parking system that allocates resource based on driver's cost function [11]. A coverage control and coordination algorithm to allocate groups of vehicles is proposed by [7]. Pavone et.al propose control methods for mobility-ondemand systems [15], [18]. Miao et al. show the advantage of looking-ahead at future demand when making a current decision, while considering to balance taxi supply throughout the city and reduce idle cruising cost [13]. These works

This work was supported by NSF CPS-1239152, Project Title: CPS: Synergy: Collaborative Research: Multiple-Level Predictive Control of Mobile Cyber Physical Systems with Correlated Context.Shuo Han was supported in part by the NSF (CNS-1239224) and TerraSwarm, one of six centers of STARnet, a Semiconductor Research Corporation program sponsored by MARCO and DARPA. F. Miao, S. Han and G. J. Pappas are with the Department of Electrical and Systems Engineering, University of Pennsylvania, Philadelphia, PA, USA 19014. Email: \{miaofei, hanshuo, pappasg\}@seas.upenn.edu. S. Lin is with Department of Electrical and Computer Engineering, Stony Brook University, Long Island, NY, USA 11794. Email:\{shan.x.lin@stonybrook.edu\}. heavily rely on precise passenger demand models to make dispatch decisions. However, the passenger demand models have their intrinsic model uncertainties, which are imposed by many factors, such as weather, working schedule, city events etc. Algorithms that do not consider these uncertainties can lead to inefficient dispatch services, resulting in long waiting times of underserved passengers, imbalanced workloads, and increased idle mileages of taxis. Therefore, it is essential to address such model uncertainties while considering performance requirements of the taxi network.

In this work, we formulate taxi dispatch problems considering polytope uncertainty demand models and two objectives. These objectives are reducing current and future expected idle cruising distance and balancing workload of taxis in each region of the entire city. Since considering anticipated future demand for making current decisions benefits taxi network performance [13], [18], while demand is affected by various factors with learnable variance [14], a dispatch approach robust to demand uncertainties is important. Different uncertainty sets for linear robust optimization problems are constructed and compared [9]. The objective function we design is convex but nonlinear over the variables and concave over the uncertain sets, which has not been covered by previous work [3], [8], [9]. We derive an equivalent convex optimization form via strong duality and minimax theorem for the robust problem to guarantee computational tractability. With the information of model uncertainties, the cost distribution of robust taxi dispatch solutions has a shorter tail than non-robust dispatch solutions.

The contributions of this work are several folds. First, we formulate a robust optimization modeling framework for studying the problem of balancing taxi supply with least idle cruising mileage under demand uncertainties. Second, we show that the multi-stage robust optimization problem has an explicit computationally tractable form and can be solved efficiently. Third, we implement the approach designed in this work and show the advantage of a robust taxi dispatch framework based on a real data set.

The rest of the paper is organized as follows. The taxi dispatch problem is described and formulated as robust optimizations in Section II, followed by equivalent computationally tractable forms in Section III. Simulation results based on a real data set is shown in Section IV. Concluding remarks are provided in Section V.

\section{PRoblem Formulation}

\section{A. Problem Overview}

The goal of taxi dispatch is to direct vacant taxis towards current and future passengers with minimum total idle 
mileage. There are two objectives. One is sending more taxis for more requests to reduce mismatch between supply and demand across all regions in the city. The other is to reduce the total idle driving distance for picking up expected passengers in order to save cost. Involving predicted customer demand of the future when making current decisions benefits to increasing total profits, since drivers are able to travel to regions with better chances to pick up future passengers.

The main problem we consider in this work is how to dispatch taxis with uncertainties in the predicted spatiotemporal patterns of passenger demand. A typical monitoring and taxi network control infrastructure is shown in Figure 1. The dispatch center periodically collects and stores real-time information such as GPS location, occupancy status and road conditions. Dispatch solutions are sent to the communication component of each taxi via cellular radio.

\section{B. Taxi network model}

We discretize time and space in problem formulation for computational efficiency. We assume that the entire city is divided into $n$ regions, and discrete time slots are indexed by $k=1,2, \ldots, \tau$. Typically, it is difficult to predict a deterministic value of passenger demand of a region during specific time. With prior knowledge and data sets, we assume that the passenger demand model is described by uncertainty vectors belonging to a polytope defined as $r^{k} \in \Delta \subset \mathbb{R}_{+}^{n}, \quad k=1, \ldots, \tau$

where $\Delta$ is a closed set and a convex polytope, and $r_{j}^{k}$ is the number of total requests within region $j$ during time $k$. The number of vacant taxis before dispatching at region $j$ is provided by real-time GPS position and occupancy information, and denoted by $L_{j}^{1}$, and $L^{1} \in \mathbb{R}_{+}^{n}$.

The problem is illustrated in Figure 2 for a box type of polytope $\Delta$, where $r_{j}^{k} \in\left[R_{1 j}^{k}, R_{2 j}^{k}\right], j=1, \ldots, n$ is the range of requests number within region $j$ during time $k$. The edge weight of the graph represents the distance between two regions. Specifically, each region has a predicted range of requests and an initial number of vacant taxis provided by real-time sensing information. The objective is to calculate the number of taxis dispatched to each region according to control requirements and constraints.

\section{Robust taxi dispatch problem formulation}

We define the dispatch order matrix as a non-negative variable matrix $X^{k} \in \mathbb{R}_{+}^{n \times n}, \quad X_{i j}^{k} \geq 0$, where $X_{i j}^{k}$ is the number of taxis dispatched from region $i$ to region $j$. Here we

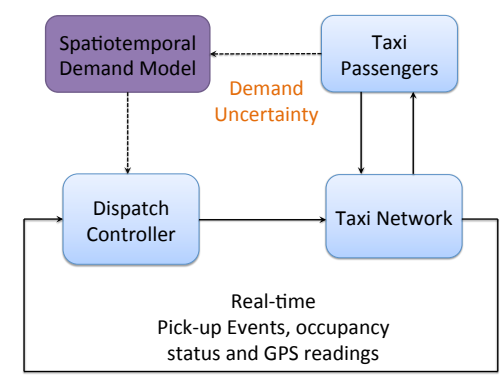

Fig. 1. A prototype of the taxi dispatch system

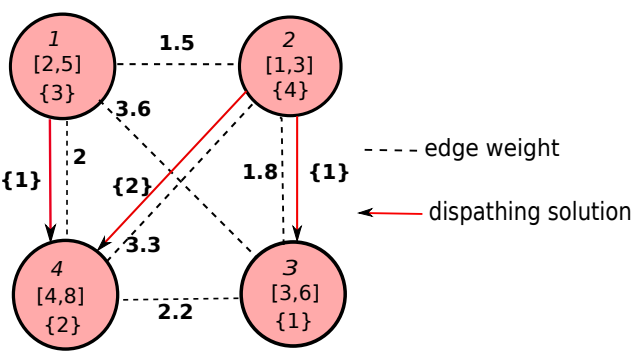

Fig. 2. Uncertain demand, vacant taxis at different regions and a dispatch solution. A circle represents a region, with a range of predicted requests $([\cdot]$ inside the circle) and a number of vacant taxis $(\{\cdot\}$ in the circle) before dispatching.

relax the integer constraint of $X_{i j}^{k} \in \mathbb{N}$ to non negative, since mixed integer programming is not computational efficient for a large-scale robust optimization problem.

Estimated across-region idle-driving distance: When traversing from region $i$ to region $j$, taxi drivers take the cost of cruising on the road without picking up a passenger till the target region. Hence, we consider to minimize this kind of idle driving distance while dispatching taxis. We define the weight matrix of the network in Fig. 2 as $W \in \mathbb{R}^{n \times n}$, where $W_{i j}$ is the distance between region $i$ and region $j$. The across-region idle driving cost according to $X^{k}$ is

$$
J_{D}\left(X^{k}\right)=\sum_{i} \sum_{j} X_{i j}^{k} W_{i j} .
$$

We assume that the region division method is time-invariant in this work, and $W$ is a constant matrix for the optimization problem formulation - for instance, the value of $W_{i j}$ represents the length of shortest path on streets from the center of region $i$ to the center of region $j$. For control algorithms with a dynamic region division method, the distance matrix can be generalized to a time dependent matrix $W^{k}$ as well.

The distance every taxi can drive should be bounded by a threshold parameter $m \in \mathbb{R}^{+}$during limited time which is equivalent to

$$
X_{i j}^{k}=0 \text { if } W_{i j}>m,
$$

$$
X_{i j}^{k} \geqslant 0, \quad X_{i j}^{k} W_{i j} \leq m X_{i j}^{k}, \quad \forall i, j \in\{1, \ldots, n\} .
$$

To explain this, assume the constraint (2) holds. If $W_{i j}>m$ and $X_{i j}^{k}>0$, we have $X_{i j}^{k} W_{i j}>m X_{i j}^{k}$, which contradicts to (2). The threshold $m$ is related to the length of time slot and traffic conditions on streets. For instance, with the same average speed of cars in one city, the value of $m$ for 20 minutes can be one third of the value of $m$ for one hour.

Metric of serving quality: We design the metric of service quality as a function $J_{E}\left(X^{k}, r^{k}\right)$ concave in $r^{k}$ and convex in $X^{k}$ in this work for computational efficiency [3]. Besides vacant taxis traverse to region $j$ according to matrix $X^{k}$, we define $L_{j}^{k} \in \mathbb{R}_{+}$as the number of vacant taxis at region $j$ before dispatching at the beginning of time $k$, and $L^{k} \in \mathbb{R}_{+}^{n}$. The total number of vacant taxis at region $i$ during time $k$ is

$$
\mathbf{1}_{n}^{T} X_{\cdot i}^{k}-X_{i}^{k} \mathbf{1}_{n}+L_{i}^{k}>0, \quad i=1, \ldots, n,
$$

where $X_{\cdot i}^{k}$ is the $i$-th column of $X^{k}$ and $X_{i}^{k}$ is the $i$-th row of $X^{k}$. One service metric is fairness at every region, or the demand and supply ratio of each region equals to that of the whole city. With balanced distribution of vacant 


\begin{tabular}{|c|c|}
\hline Parameters & Description \\
\hline$n$ & the number of regions \\
\hline$\tau$ & model predicting time horizon \\
\hline$r^{k} \in \Delta \subset \mathbb{R}_{+}^{n}, \Delta$ is a polytope & weight matrix, $w_{i j}$ is the distance from region $i$ to region $j$ \\
\hline$W \in \mathbb{R}^{n \times n}$ & probability matrix that describes taxi mobility patterns during one time slot \\
\hline$P^{k} \in[0,1]^{n \times n}$ & the initial number of vacant taxis at each region provided by GPS and occupancy status data \\
\hline$L^{1} \in \mathbb{N}^{n}$ & the upper bound of distance each taxi can drive idly for picking up a passenger \\
\hline$m \in \mathbb{R}^{+}$ & the weight factor of the objective function \\
\hline$\alpha \in \mathbb{R}_{+}$ & Description \\
\hline$\beta \in \mathbb{R}_{+}$ & the number of taxis dispatched from region $i$ to region $j$ during time $k$ \\
\hline$X_{i j}^{k} \in \mathbb{R}_{+}$ & the number of vacant taxis at each region before dispatching at the beginning of time $k$ \\
\hline$L^{k} \in \mathbb{R}_{+}^{n}$ &
\end{tabular}

TABLE I

PARAMETERS AND VARIABLES OF TAXI DISPATCH PROBLEM (9).

taxis, system performance is good from the perspective that a customer's expected waiting time is small as shown by a queueing theoretic model in [18]. Intuitively, the objective of balanced demand and supply ratio is to minimize

$$
\sum_{k=1}^{\tau} \sum_{i=1}^{n}\left|\frac{r_{i}^{k}}{\mathbf{1}_{n}^{T} X_{\cdot i}^{k}-X_{i}^{k} \mathbf{1}_{n}+L_{i}^{k}}-\frac{\mathbf{1}_{n}^{T} r^{k}}{N}\right| .
$$

However, function (4) is not concave of $r^{k}$, a robust optimization problem with uncertain parameter $r^{k}$ is not able to be converted to an equivalent computationally tractable convex form. Hence, we design a surrogate function for (4)

$$
J_{E}\left(X^{k}, r^{k}\right)=\sum_{i} \frac{r_{i}^{k}}{\left(\mathbf{1}_{n}^{T} X_{\cdot i}^{k}-X_{i}^{k} \mathbf{1}_{n}+L_{i}^{k}\right)^{\alpha}},
$$

with $\alpha \rightarrow 0$. The function $J_{E}\left(X^{k}, r^{k}\right)$ is affine in $r^{k}$ for any $X^{k}$, and convex in $X^{k}$ for any $r^{k}$, since $\mathbf{1}_{n}^{T} X_{\cdot i}^{k}-X_{i}^{k} \mathbf{1}_{n}+$ $L_{i}^{k}>0$. Now we explain why function $J_{E}$ (5) with $\alpha \rightarrow 0$ is a surrogate function of (4) when we consider to minimize the value of (4) under certain constraints.

Let $N$ be the total number of vacant taxis in the whole city before dispatching for time $k$. The number does not change after dispatching before taxis picking up passengers, i.e.

$$
\sum_{i}\left(\mathbf{1}_{n}^{T} X_{\cdot i}^{k}-X_{i}^{k} \mathbf{1}_{n}+L_{i}^{k}\right)=\sum_{i} L_{i}^{k}=N .
$$

Consider the following problem

$$
\underset{b>0, \sum_{i} b_{i}=c}{\operatorname{minimize}} \sum_{i} \frac{a_{i}}{b_{i}^{\alpha}}, c \text { is a constant. }
$$

Substitute $b_{n}=c-b_{1} \cdots-b_{n-1}$ into (6), and take partial derivatives of $\sum_{i} \frac{a_{i}}{b_{i}^{\alpha}}$ over $b_{i}, i=1, \ldots, n-1$. To get the minimum, each partial derivative should be 0 , namely

which is equivalent to

$$
-\alpha \frac{a_{i}}{b_{i}^{\alpha+1}}-\alpha(-1) \frac{a_{n}}{\left(c-b_{1} \cdots-b_{n-1}\right)^{\alpha+1}}=0,
$$

$$
\frac{a_{1}}{b_{1}^{\alpha+1}}=\cdots=\frac{a_{n-1}}{b_{n-1}^{\alpha+1}}=\frac{a_{n}}{b_{n}^{\alpha+1}} .
$$

Hence, when $\alpha \rightarrow 0, \alpha+1 \stackrel{n}{\rightarrow}$, the optimal solution of minimizing $J_{E}$ over $X^{k}$ satisfies

$$
\frac{r_{j}}{\mathbf{1}_{n}^{T} X_{\cdot j}^{k}-X_{j}^{k} \mathbf{1}_{n}+L_{j}^{k}}=\frac{\mathbf{1}_{n}^{T} r^{k}}{N}
$$

The number of initial vacant taxis at time $k+1$ depends on the mobility patterns of passengers during time $k$, which we do not directly control. Similarly as a queueing theoretic model, we define $P_{i j}^{k}$ as the probability that a vacant taxi traverses from region $i$ to region $j$ during time $k$ and turns to an available supply for time $k+$ 1. One example of $P_{i j}^{k}$ based on a data set is the taxi mability pattern defined in work [13]. Then the number of vacant taxis within region $j$ by the end of time $k$ is

$$
\left(\mathbf{1}_{n}^{T} X^{k}-\left(X^{k} \mathbf{1}_{n}\right)^{T}+\left(L^{k}\right)^{T}\right) P_{\cdot j}^{k},
$$

where $P_{\cdot j}^{k}$ is the $j$-th column of $P^{k}$, and

$$
\left(L^{k+1}\right)^{T}=\left(\mathbf{1}_{n}^{T} X^{k}-\left(X^{k} \mathbf{1}_{n}\right)^{T}+\left(L^{k}\right)^{T}\right) P^{k} .
$$

Multi-objective function: Since there exists a trade-off between two objectives, we define a weight parameter $\beta$ of wo objectives $J_{D}\left(X^{k}\right)$ in (1) and $J_{E}\left(X^{k}, r^{k}\right)$ in (5). Without considering model uncertainties corresponding to $r^{k}$, a convex optimization form of taxi dispatch problem is

$$
\begin{aligned}
& \min _{X^{1}, \ldots, X^{\tau}, L^{2}, \ldots, L^{\tau}} \quad J=\sum_{k=1}^{\tau}\left(J_{D}\left(X^{k}\right)+\beta J_{E}\left(X^{k}, r^{k}\right)\right) \\
& =\sum_{k=1}^{\tau} \sum_{i}\left(\sum_{j} X_{i j}^{k} W_{i j}+\frac{\beta r_{i}^{k}}{\left(\mathbf{1}_{n}^{T} X_{\cdot i}^{k}-X_{i}^{k} \mathbf{1}_{n}+L_{i}^{k}\right)^{\alpha}}\right) \\
& \text { s.t. (2), (3), (7). }
\end{aligned}
$$

Robust taxi dispatch problem formulation: We aim to find out a dispatch solution robust to disturbance factors of demand model in this work. While considering future demand benefits for reducing idle driving distance as shown in [13], model uncertainties affect the decisions and we consider to minimize the cost of the worst-case scenario. For time $k=1, \ldots, \tau$, uncertain demand $r^{k}$ only affects the dispatch solutions of $k+1, \ldots, \tau$, similar to the multistage robust optimization problem in [5]. Hence, with a list of parameters and variables shown in Table I, when considering profits of future picking up decisions, a multi-stage robust taxi dispatch problem is defined as following

$$
\begin{aligned}
\min _{X^{1}} . & \max _{r^{1} \in \Delta} \min _{X^{2}, L^{2}} \max _{r^{2} \in \Delta} \ldots \min _{X^{\tau}, L^{\tau}} \max _{r^{\tau} \in \Delta} \\
J= & \sum_{k=1}^{\tau}\left(J_{D}\left(X^{k}\right)+\beta J_{E}\left(X^{k}, r^{k}\right)\right) \\
= & \sum_{k=1}^{\tau} \sum_{i}\left(\sum_{j} X_{i j}^{k} W_{i j}+\frac{\beta r_{i}^{k}}{\left(\mathbf{1}_{n}^{T} X_{\cdot i}^{k}-X_{i}^{k} \mathbf{1}_{n}+L_{i}^{k}\right)^{\alpha}}\right) \\
\text { s.t. } & \left(L^{k+1}\right)^{T}=\left(\mathbf{1}_{n}^{T} X^{k}-\left(X^{k} \mathbf{1}_{n}\right)^{T}+\left(L^{k}\right)^{T}\right) P^{k}, \\
& \mathbf{1}_{n}^{T} X^{k}-\left(X^{k} \mathbf{1}_{n}\right)^{T}+\left(L^{k}\right)^{T}>0, \\
& X_{i j}^{k} W_{i j} \leq m X_{i j}^{k}, \\
& X_{i j}^{k} \geq 0, \quad i, j \in\{1,2, \ldots, n\} .
\end{aligned}
$$


After getting an optimal solution $X^{1}$ of (9), we adjust the solution by rounding methods to get an integer number of taxis to be dispatched towards corresponding regions. A feasible integer solution of (9) always exists, since $X_{i j}^{k}=0, \forall i, j, k$ is feasible. For a special case $\tau=1$, the constraint (7) does not exist any more, other constraints and objective of (9) are adjusted to the form of $\tau=1$.

\section{Computationally Tractable Formulations}

We build equivalent convex optimization formulations of problem (9) with different definitions of polytope uncertainty sets in this section, and show that the robust taxi dispatch problem in this work is computationally tractable. Robust linear programming problems with ellipsoid uncertainties are discussed in [3]. The authors reformulate constraints of the original problem to equivalent convex constraints that must hold for the uncertainty sets. The form of nonlinear objective function designed in problem (9) is not covered by previous work like [3].

We derive the computationally tractable form of one-stage robust optimization (9) in the following theorem.

Theorem 1: (Next Step Dispatch) When $\tau=1$, and the uncertain parameter set $r \in \Delta$ of problem (9) is defined as a polytope with the form $\Delta:=\{r \geq 0, A r \leq b\}$, problem (9) with $\tau=1$ is equivalent to the following convex optimization problem (we omit the superscripts $k$ for variables and parameters without confusion)

$$
\begin{aligned}
& \underset{X \geq 0, \lambda \geq 0}{\operatorname{minimize}} \sum_{i} \sum_{j} X_{i j} W_{i j}+b^{T} \lambda \\
& \text { subject to } A^{T} \lambda-\beta\left[\begin{array}{c}
\frac{1}{\left(\mathbf{1}_{n}^{T} X_{\cdot 1}-X_{1} \cdot \mathbf{1}_{n}+L_{1}\right)^{\alpha}} \\
\vdots \\
\frac{1}{\left(\mathbf{1}_{n}^{T} X_{\cdot n}-X_{n} \cdot \mathbf{1}_{n}+L_{n}\right)^{\alpha}}
\end{array}\right] \geq 0 \text {, }
\end{aligned}
$$

(2), (3).

Proof: For any fixed $X$, the maximum part of the objective function is equivalent to

$$
\begin{aligned}
\max _{r \in \Delta} & f(X, r)+d(X)=c^{T}(X) r+d(X) \\
= & \beta \sum_{i} \frac{r_{i}}{\left(\mathbf{1}_{n}^{T} X_{\cdot i}-X_{i} \cdot \mathbf{1}_{n}+L_{i}\right)^{\alpha}}+\sum_{i} \sum_{j} X_{i j} W_{i j}, \\
c(X)_{i}= & \beta \frac{1}{\left(\mathbf{1}_{n}^{T} X_{\cdot i}-X_{i} \cdot \mathbf{1}_{n}+L_{i}\right)^{\alpha}}, d(X)=\sum_{i} \sum_{j} X_{i j} W_{i j} .
\end{aligned}
$$

The Lagrangian of problem (11) with the Lagrangian multipliers $\lambda \geq 0, v \geq 0$ is

$$
\begin{aligned}
\mathcal{L}(X, r, \lambda, v) & =c^{T}(X) r+d(X)-\lambda^{T}(A r-b)+v^{T} r, \\
& =d(X)+b^{T} \lambda-\left(A^{T} \lambda-c(X)-v\right)^{T} r,
\end{aligned}
$$

where $\left(A^{T} \lambda-c(X)-v\right)^{T} r$ is linear of $r$, and the upper bound exists only when $A^{T} \lambda-c(X)-v=0$, or $A^{T} \lambda-c(X) \geqslant 0$. The objective function of the dual problem is

$$
\begin{aligned}
g(X, \lambda, v) & =\sup _{r \in \Delta} \mathcal{L}(X, r, \lambda, v) \\
& =\left\{\begin{array}{l}
d(X)+b^{T} \lambda \text { if } A^{T} \lambda-c(X) \geqslant 0 . \\
\infty \quad \text { otherwise }
\end{array}\right.
\end{aligned}
$$

Strong duality holds for problem of (11) since it is convex, and its dual problem is

$$
\begin{array}{ll}
\underset{\lambda \geq 0}{\operatorname{minimize}} & d(X)+b^{T} \lambda \\
\text { subject to } & A^{T} \lambda-c(X) \geq 0 .
\end{array}
$$

Hence, problem (9) with $\tau=1$ can be solved as the convex optimization problem defined in (10).

For the multi-stage robust optimization problem (9), the computationally tractable convex form depends on the definition of uncertainty sets. An approximated semidefinite programming form for calculating time dependent control input of linear dynamical systems affected by uncertainty is proposed in [5]. We consider both time independent and time dependent polytope uncertainties exist in this work.

When the demand uncertainty sets during $k=1, \ldots, \tau$ are independent, the process of converting (9) to an equivalent convex form is similar to that of the one-stage robust optimization problem and described as the following lemma.

Lemma 1: If the uncertain parameter sets for $r^{k}, k=$ $1, \ldots, \tau$ are independent, defined as polytopes with the form

$$
\Delta_{k}:=\left\{r^{k} \geq 0, A_{k} r^{k} \leq b_{k}\right\}, \quad k=1, \ldots, \tau,
$$

problem (9) is equivalent to the following convex optimization problem

$$
\begin{array}{ll}
\min _{X^{k}, L^{k}, \lambda^{k} \geq 0} & \sum_{k=1}^{\tau}\left(\sum_{i} \sum_{j} X_{i j}^{k} W_{i j}+b_{k}^{T} \lambda^{k}\right) \\
\text { subject to } & A_{k}^{T} \lambda^{k}-\beta\left[\begin{array}{c}
\frac{1}{\left(\mathbf{1}_{n}^{T} X_{\cdot 1}^{k}-X_{1}^{k} \mathbf{1}_{n}+L_{1}^{k}\right)^{\alpha}} \\
\vdots \\
\frac{1}{\left(\mathbf{1}_{n}^{T} X_{\cdot n}^{k}-X_{n}^{k} \cdot \mathbf{1}_{n}+L_{n}^{k}\right)^{\alpha}}
\end{array}\right] \geq 0,
\end{array}
$$

other constraints of (9), $k=1, \ldots, \tau$

Proof: When the uncertainty set for each $r^{k}$ are independent defined as (15), the objective function and constraint of problem (9) are separable over $k$, except the constraint of $L^{k+1}$ (7). Noting that $L^{k+1}$ in (7) is irrelevant to $r^{k}$ given $X^{1}, \ldots, X^{k-1}$ and $L^{1}, \ldots, L^{k-1}$, and it does not affect the process of deriving the dual problem of maximize the objective function over $r^{k}$. The objective function of multistage problem (9) is then equivalent to

$$
\min _{X^{1}} \max _{r^{1} \in \Delta_{1}} J_{1}+\sum_{k=2}^{\tau} \min _{X^{k}, L^{k}} \max _{r^{k} \in \Delta_{k}} J_{k}=\min _{X^{k}, L^{k} r^{k} \in \Delta_{k}} \max _{k=1}^{\tau} J_{k}
$$

The Lagrangian of (17) with multipliers $\lambda^{k} \geq 0, v^{k} \geq 0$ is

$$
\begin{aligned}
& \mathcal{L}\left(X^{k}, r^{k}, \lambda^{k}, v^{k}\right) \\
= & \sum_{k=1}^{\tau}\left(d\left(X^{k}\right)+b_{k}^{T} \lambda^{k}-\left(A_{k}^{T} \lambda^{k}-c\left(X^{k}\right)-v^{k}\right)^{T} r^{k}\right),
\end{aligned}
$$

Based on the proof of Theorem 1, we take partial derivative of the Lagrangian (18) for every $r^{k} \in \Delta_{k}$, group all the other constraints and get the dual form defined as (16). 
The following theorem describes the equivalent computationally tractable form of (9) for a more general case when demand uncertainty sets are spatial temporal correlated.

Theorem 2: When the uncertainty set for $r^{1}, \ldots, r^{\tau}$ are correlated, each $r^{k}$ belongs to a compact set, and $\Delta$ satisfies

$$
\Delta:=\left\{A_{1} r^{1}+\cdots+A_{\tau} r^{\tau} \leq b, r^{k} \geq 0\right\},
$$

problem (9) is equivalent to a convex optimization problem

$$
\begin{array}{lc}
\min _{X^{k}, L^{k}, \lambda \geq 0} & \sum_{k=1}^{\tau}\left(\sum_{i} \sum_{j} X_{i j}^{k} W_{i j}\right)+b^{T} \lambda \\
\text { subject to } & A_{k}^{T} \lambda-\beta\left[\begin{array}{c}
\frac{1}{\left(\mathbf{1}_{n}^{T} X_{\cdot 1}^{k}-X_{1}^{k} \mathbf{1}_{n}+L_{1}^{k}\right)^{\alpha}} \\
\vdots \\
\frac{1}{\left(\mathbf{1}_{n}^{T} X_{. n}^{k}-X_{n}^{k} \cdot \mathbf{1}_{n}+L_{n}^{k}\right)^{\alpha}}
\end{array}\right] \geq 0,
\end{array}
$$

constraints of (9), $k=1, \ldots, \tau$.

Proof: With uncertain set defined as (19), $r^{1}, \ldots, r^{\tau}$ are not independent, and we first derive the Lagrangian and dual of the maximum part of the objective function (9) with constraint $\lambda \geq 0, v_{\tau} \geq 0$ for the last stage $\tau$

$$
\begin{aligned}
& \mathcal{L}_{\tau}\left(X^{k}, r^{k}, \lambda, v_{\tau}\right) \\
= & \sum_{k=1}^{\tau}\left(c^{T}\left(X^{k}\right) r^{k}+d\left(X^{k}\right)\right)+v_{\tau}^{T} r^{\tau}-\lambda^{T}\left(A_{1} r^{1}+\cdots+A_{\tau} r^{\tau}-b\right), \\
= & b^{T} \lambda-\sum_{k=1}^{\tau}\left(\left(A_{k}^{T} \lambda-c\left(X^{k}\right)\right)^{T} r^{k}-d\left(X^{k}\right)\right)-v_{\tau}^{T} r^{\tau},
\end{aligned}
$$

Taking the partial derivative of the above equation over $r^{\tau}$, similarly as the proof of Theorem 1 , the objective function of the dual problem is

$$
\begin{aligned}
& g_{\tau}\left(X^{k}, \lambda, r^{1}, \ldots, r^{\tau-1}\right)=\sup _{r^{T} \in \Delta} \mathcal{L}_{\tau}\left(X^{k}, r^{k}, \lambda, v_{\tau}\right) \\
= & \left\{\begin{array}{l}
\infty \text { if } A_{\tau}^{T} \lambda-c\left(X^{\tau}\right)<0, \\
\tau-1 \\
\sum_{k=1}^{\tau-1}-\left(A_{k}^{T} \lambda-c\left(X^{k}\right)\right)^{T} r^{k}+\sum_{k=1}^{\tau} d\left(X^{k}\right)+b^{T} \lambda \quad \text { o.w. }
\end{array}\right.
\end{aligned}
$$

Problem (9) is equivalent to the following form without $r^{\tau}$

$$
\begin{aligned}
\min _{X^{1}} \cdot \max _{r^{1} \in \Delta} \min _{X^{2}, L^{2}} \max _{r^{2} \in \Delta} \ldots \max _{r^{\tau-1} \in \Delta} \min _{X^{\tau}, L^{\tau}, \lambda} \\
\quad \sum_{k=1}^{\tau-1}-\left(A_{k}^{T} \lambda-c\left(X^{k}\right)\right)^{T} r^{k}+\sum_{k=1}^{\tau} d\left(X^{k}\right)+b^{T} \lambda
\end{aligned}
$$

s.t. $\quad A_{\tau}^{T} \lambda-c\left(X^{\tau}\right) \geq 0$ and constraints of (9).

Now consider the minimax problem over stage $\tau-1$ and $\tau$ of the above problem

$$
\begin{aligned}
& \max _{r^{\tau-1} \in \Delta} \min _{X^{\tau}, L^{\tau}, \lambda} \\
& \quad \sum_{k=1}^{\tau-1}-\left(A_{k}^{T} \lambda-c\left(X^{k}\right)\right)^{T} r^{k}+\sum_{k=1}^{\tau} d\left(X^{k}\right)+b^{T} \lambda
\end{aligned}
$$

s.t. $\quad A_{\tau}^{T} \lambda-c\left(X^{\tau}\right) \geq 0$ and constraints of (9).

The Domain of problem (21) satisfies that $X^{\tau}, L^{\tau}, \lambda$ is compact (convex and closed), $r^{\tau-1}$ is compact. The objective function is a closed function convex over $X^{\tau}, L^{\tau}, \lambda$ and concave over $r^{\tau-1}$. According to Proposition 2.6 .9 with condition (1) of book [4], the set of saddle points of (21) is nonempty, and we can exchange the order of max and min without changing the optimal solution:

$$
\max _{r^{\tau-1} \in \Delta} \min _{X^{\tau}, L^{\tau}, \lambda} g_{\tau}=\min _{X^{\tau}, L^{\tau}, \lambda} \max _{r^{\tau-1} \in \Delta} g_{\tau}
$$

Then we derive the dual form for stage $\tau-1$ with respect to maximum over $r^{\tau-1}$, and only one additional constraint $r^{\tau-1} \geq 0$ with corresponding multiplier $v^{\tau-1}$ is added to the Lagrangian of stage $\tau-1$ based on $g_{\tau}$ :

$$
\begin{aligned}
& \mathcal{L}_{\tau-1}\left(X^{1}, r^{1}, \ldots, X^{\tau-1}, r^{\tau-1}, \lambda, v_{\tau-1}\right) \\
= & \sum_{k=1}^{\tau-1}\left(c\left(X^{k}\right)-A_{k}^{T} \lambda\right)^{T} r^{k}+\sum_{k=1}^{\tau} d\left(X^{k}\right)+b^{T} \lambda-v_{\tau-1}^{T} r^{\tau-1} .
\end{aligned}
$$

Similarly, we calculate partial derivative of $\mathcal{L}_{\tau-1}$ over $r^{\tau-1}$ to get $g_{\tau-1}$, and exchange the order of $\max$ and $\min$ in the objective function. By induction to $k=1$, we have the following convex optimization form of the objective function for formulation (9)

$$
\underset{X^{k} \geq 0, L^{k} \geq 0, \lambda \geq 0}{\operatorname{minimize}} \sum_{k=1}^{\tau}\left(\sum_{i} \sum_{j} X_{i j}^{k} W_{i j}\right)+b^{T} \lambda .
$$

Hence, with all the constraints, problem (20) is the computationally tractable convex optimization form of problem (9) under dependent uncertain set (19).

With equivalent convex optimization forms under different uncertainty sets, robust taxi dispatch problem (9) is computationally tractable and solved efficiently.

\section{Simulations}

We conduct simulations based on a San Francisco taxi data set [16]. Information for each individual taxi includes three components: the Unix epoch time, the geometric position (latitude and longitude), and a binary indicator of whether the taxi is vacant or with passengers. We show the motivation to find robust dispatch solutions with model uncertainties, and compare the optimal cost of robust dispatch (9) with convex optimization form (8) in this section.

Estimate uncertainty sets for demand $r^{k}$ :

A boxplot of total number of requests (pick up events) during one hour $(5: 00-6: 00 \mathrm{pm})$ in different regions is shown in Figure 3. The mean and standard deviation of the model are calculated via bootstrap [6]. Figure 3 shows a motivation of this work - a robust dispatch algorithm to

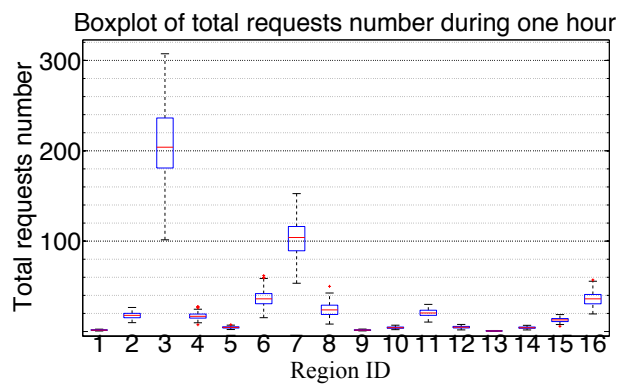

Fig. 3. Boxplot of total number of equests at each region during one hour The red line in the middle shows the median value of all samples, the box shows the distribution of data, with range first quartile and third quartile. 


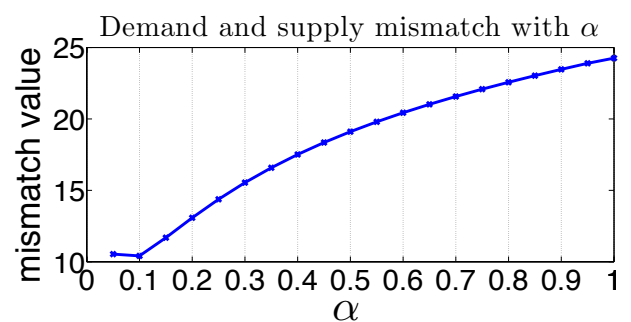

Fig. 4. Comparison of demand and supply mismatch values defined as (4) with different solutions for minimizing $J_{E}$ defined in (5) with $\alpha$ in range $(0,1]$. The value of function (4) under an optimal solution of $J_{E}$ is smaller with an $\alpha$ closer to 0 , which means the dispatch solution tends to be more balanced throughout the entire city.

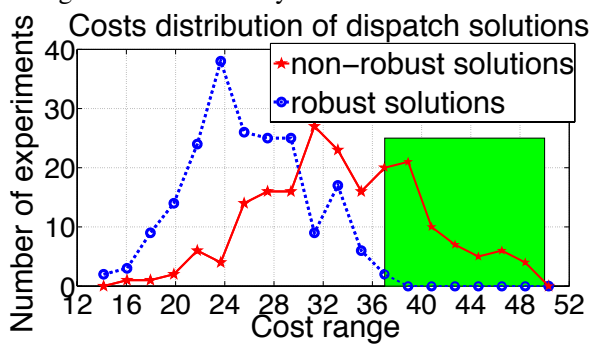

Fig. 5. Cost distribution comparison of robust optimization (9) solutions in this work and non-robust optimization (8) solutions. The lines show the number of experiments with cost falling in intervals $[12,14],(14,16], \ldots,(48,50]$ of two methods applying Monte-Carlo experiments based on the historical data set. Robust optimization solutions in this work has a shorter tail than non-robust solutions.

balance the number of taxis according to the demand from the perspective of system-level optimal performance.

How vacant taxis are balanced across regions with different $\alpha$ values: Figure 4 shows mismatch between supply and demand defined as equation (4) for different optimal solutions of minimizing $J_{E}$ defined in (5) for $\alpha \in(0,1]$. With $\alpha$ closer to 0 , the optimal value of (4) is smaller. We choose $\alpha=0.1$ for calculating optimal solutions of (9) and (8) in this section.

Compare robust solutions with non-robust solutions: We compare the cost distribution of 200 Monte-Carlo simulations based on the data set of robust optimization solutions (9) and convex optimization solutions (8) in Figure 5. The customer demand models applied in the two algorithms are different. For the objective function (8), the nominated demand prediction $r^{k}$ is a deterministic value, for instance - the average or mean of the bootstrap model which is constructed based on the historical data set. For the robust problem formulation (9) considered in this work, the uncertainty set is a box defined according to the mean and covariance matrix of the bootstrap model.

Figure 5 shows that the robust dispatch solutions result in $35.5 \%$ fewer experiments with a cost greater than 37 , compared with non-robust solutions. It means the cost distribution of the robust optimization (9) in this work has a shorter tail than that of the deterministic convex optimization formulation (8). With model uncertainty information in decision making, system performance is improved compared with solutions only based on the nominal demand model.

\section{CONCLUSiON}

In this paper, we propose a novel robust optimization formulation to deal with demand model uncertainties in taxi dispatch solutions. The robust optimization problem formulation is constructed based on polytopic type of model uncertainties, considering multiple objectives and practical constraints. Transformed to an equivalent convex optimization form, the problem is solvable in polynomial time. By experimenting the method in this work on a real historical GPS and occupancy record data set, we show that robust dispatch solutions are less probably to have relatively large costs. In the future, we will enhance problem formulation considering more uncertain characteristics of taxi network model, like passenger destinations and road congestions.

\section{REFERENCES}

[1] M. Asif, J. Dauwels, C. Goh, A. Oran, E. Fathi, M. Xu, M. Dhanya, N. Mitrovic, and P. Jaillet. Spatio and temporal patterns in largescale traffic speed prediction. IEEE Transactions on Intelligent Transportation Systems, (15):797-804, 2014.

[2] R. K. Balan, K. X. Nguyen, and L. Jiang. Real-time trip information service for a large taxi fleet. In Proceedings of the 9th International Conference on Mobile Systems, Applications, and Services, MobiSys '11, pages 99-112. ACM, 2011.

[3] A. Ben-Tal and A. Nemirovski. Robust convex optimization. Mathematics of Operations Research, 23(4):769-805, 1998.

[4] D. Bertsekas, A. Nedi, A. Ozdaglar, et al. Convex Analysis and Optimization. Athena Scientific, 2003.

[5] D. Bertsimas, D. Iancu, and P. Parrilo. A hierarchy of near-optimal policies for multistage adaptive optimization. IEEE Transactions on Automatic Control, 56(12):2809-2824, Dec 2011.

[6] E. Bradley. Bootstrap methods: Another look at the jackknife. The Annals of Statistics, 7(1-26), 1979.

[7] J. Cortes, S. Martinez, T. Karatas, and F. Bullo. Coverage control for mobile sensing networks. IEEE Transactions on Robotics and Automation, 20(2):243-255, 2004.

[8] F. A. Cuzzola, J. C. Geromel, and M. Morari. An improved approach for constrained robust model predictive control. Automatica, 38(7):1183-1189, 2002.

[9] B. Dimitris, B. Vishal, and K. Nathan. Data-driven robust optimization. Operations Research, (arXiv: 1401.0212).

[10] R. Ganti, M. Srivatsa, and T. Abdelzaher. On limits of travel time predictions: Insights from a new york city case study. In 2014 IEEE 34th International Conference on Distributed Computing Systems (ICDCS), pages 166-175, June 2014.

[11] Y. Geng and C. Cassandras. New "smart parking" system based on resource allocation and reservations. IEEE Transactions on Intelligent Transportation Systems, 14(3):1129-1139, 2014.

[12] J. Herrera, D. Work, R. Herring, X. Ban, Q. Jacobson, and A. Bayen. Evaluation of traffic data obtained via GPS-enabled mobile phones: The Mobile Century field experiment. Transportation Research Part C, 18(4):568-583, 2010.

[13] F. Miao, S. Lin, S. Munir, J. A. Stankovic, H. Huang, D. Zhang, T. He, and P. G. J. Taxi dispatch with real-time sensing data in metropolitan areas - a receding horizon control approach. In 6th International Conference of Cyber-Physical Systems, 2015.

[14] L. Moreira-Matias, J. Gama, M. Ferreira, J. Mendes-Moreira, and L. Damas. Predicting taxi-passenger demand using streaming data. IEEE Transactions on Intelligent Transportation Systems, 14(3):13931402, Sept 2013.

[15] M. Pavone, S. L. Smith, E. Frazzoli, and R. Daniela. Robotic load balancing for mobility-on-demand systems. The International Journal of Robotics Research, 31(7):839-854, 2012.

[16] M. Piorkowski, N. Sarafijanovic-Djukic, and M. Grossglauser. A parsimonious model of mobile partitioned networks with clustering. In First International Communication Systems and Networks and Workshops (COMSNETS), pages 1-10, 2009.

[17] D. Work, S. Blandin, O. Tossavainen, B. Piccoli, and A. Bayen. A traffic model for velocity data assimilation. Applied Research Mathematics eXpress (ARMX), pages 1-35, 2010.

[18] R. Zhang and M. Pavone. Control of robotic mobility-on-demand systems: a queueing-theoretical perspective. In Proceedings of Robotics: Science and Systems, July 2014. 